\title{
Folk use of plant resource at Madi valley of Chitwan District, Nepal
}

\begin{abstract}
B.K. Bishokarma ${ }^{1}$, C.K. Kinsey ${ }^{2}$, D.R. Dangol ${ }^{3}$ and P. Chaudhary ${ }^{4}$
The purpose of this study was to compare the use of the forest plant resources and folk nomenclature in the two communities of the Madi valley a Chitwan District. The information was collected during 2001 from 83 households in Chirauli and 57 households in Dhikurbari using semi-structured questionnaires. The folk nomenclature and relative importance of the plant species for medicine, firewood, fodder and timber differ in the two communities were recorded. A total of 128 species were reported in use by both communities, out of which 64 were common to both communities. The plant species used only in Chirauli accounted for 44 and that in Dhikurbari are 20. Tharu community of Chirauli reported higher number of plant species for medicinal, fodder and forage, fuel wood and timber than that of migrated people of Dhikurbari.
\end{abstract}

\section{Key Words:}

C hitwan is a district where existences of many ethnic groups make a web and offers opportunities to exchange knowledge on use of plant resources. This include naming, use and valuation of forest tree species for different purposes has now been transferred from one community to another. Many indigenous plants have been reported for various uses by ethnic communities (Khan, 1998; Manandhar, 1990; Rijal, 1994; Shakya et al., 1995; Dangol and Gurung, 1991; Mueller- Boeker 1993). Dangol (2002) has described many forest plant species of this district and their economic value. However, the plant resources used by migrant communities, use-value, naming and relative importance and comparisons in those aspects are yet to the subject of study. Hence, an attempt has been made here to document the species diversities that are used by two different communities of the Madi valley that differ in social, economic and cultural settings. The study dug out the information on the variation in both the villages on naming, use values and valuation for different purposes.

\section{Materials and Methods}

\section{Survey site}

The study was conducted in two communities; Tharu in Chirauli and other migrants at Dhikurbari of the
Madi valley of Chitwan District. These villages differ each other in socio-economic and cultural aspects. The people rely on the forest and forest products for their social, economic and cultural requirements. Churia hill range, which is rich in forest diversity, extends across the southern frontier of Madi region and offers many useful medicine, fodder, fuel wood and timber tree species to the dependents. Traditional norms still pervail in both the villages, as the Madi area itself is not highly accessible to the big cities and market set up.

\section{Information collection}

Since the knowledge on the use of forest plant diversity is uniformly distributed among different communities within same ethnic groups, only one village each from Tharu community and hill migrant community was purposively selected. In each village, a small tole (cluster) was selected to draw sample population. All the households in the selected tole were then interviewed using semistructured questionnaires. A total of 83 households in Chirauli and 57 in Dhikurbari were surveyed, each figure representing $80 \%$ of total households in each village.

\footnotetext{
${ }^{1}$ Seed Tree Nepal, Bharatpur, Chitwan, Nepal

${ }^{2}$ Executive Director, SeedTree, Inc., USA

${ }^{3}$ Institute of Agriculture and Animal Science, Rampur, Chitwan, Nepal

${ }^{4}$ The Heller School for Social Policy and Management, Brandies University, USA
} 


\section{Results and discussion}

\section{Relative importance of plant resources in two communities}

Out of 128 plant species reported, 64 were common to both communities. Forty-four species were reported from Chirauli while 20 by Dhikurbari. During the survey, it was revealed that Neem (Azadirachta indica), Dudhkaraiya (Holarrbena antidycentrica), Jamun (Syzyzium cumini), Bel (Agle marmelos) and Med (Viscum album), were the most commonly used species for medicinal purpose in Chirauli, and those of Dhikurbari were Neem (Azadirachta indica), Hadchur (Med) (Viscum album), Dahikamle (Callicarpa macrophylla), Tulsi (Ocimum tenuiflorum) and Harro (Terminalia chebula). For fuelwood, Sal (Sorea robusta), Bakaina (Melia azedarach), Botdhairo (Lagerstromia parviflora), Sisau (Dalbergia sisoo) and Panan (Desmodium oojeinense) were most common in Chirauli, and Sindure (Mallotus phillippensis), Sal (Shorea robusta) Baira, Piyal and Botdhairo (Lageresrtomia parviflora), were the most common in Dhikurbari. However, many people put several species other than Sal in "Kukath" category (less important wood), the figures for different species might vary. Likewise, people in Chirauli valued Bakaina, Ipil- ipil (Leuceena leucocephala), Dumari (Ficus racemosa), Khanue (Ficus semicordata) and Panan for fodder, where as in Dhikurbari, Sajan (Demodium oojeinense), Galene (Leea crispa), Khannue (Ficus semicordata), Lampate (Aesculus indica) and Gayo (Bridelia retusa) were the most valued tree species for fodder purpose. For timber, Sal is the most common tree species followed by Bakaina, Sisoo, Satisal (Dalbergia latifolia) and Panan in Chirauli. In Dhikurbari, Sal is only one species that is used by large number of people followed by Bakaina, Saj (Terminalia alata), Gayo and Jamun. Results on use of each plant species for different purposes have presented in Table (2). Species use were higher for Chirauli (108) while that of Dhikurbari was 84 (Appendix 1). It revealed that the Tharu community used more plant species than did the migrant community.

\section{Multipurpose trees}

Many of the species reported in the two communities have more than one use, which further increase the importance of those species. In Chirauli, a total of 35 species are reported as multipurpose, of which Anp (Mangifera indica) and Jamun have fourfold uses, 11 species have threefold, and the rests 22 have twofold uses. In Dhikurbari, only 12 species were reported as multipurpose, of which 5 have threefold uses and rest 7 have twofold uses. Most of the multipurpose species reported in both the villages are used for firewood.

The result shows that knowledge on naming, use methods and valuation of forest plant diversity in one community differs from another community due to, in part, variation in socio-cultural, economic and political situation. Since indigenous people use greater number of forest species more commonly than migrants do, they hold better knowledge and

Table 1: Number of species reported for different purposes in Chirauli and Dhikurbari.

\begin{tabular}{lcccc}
\hline \multicolumn{1}{c}{ Communities } & Medicinal & Fodder and forage & Fuelwood & Timber \\
\hline Chirauli & $56(62.22)$ & $43(51.8)$ & $45(75)$ & $18(60.0)$ \\
Dhikurbari & $34(37.78)$ & $40(48.2)$ & $15(25)$ & $12(40.0)$ \\
\hline
\end{tabular}

Figures in the parentheses represent percentages of the total used species.

Table 2: Five most important species for each purpose based on household survey

\begin{tabular}{cllll}
\hline \multicolumn{1}{c}{ S.N. } & Medicinal & Fodder and forage & Fuelwood & Timber \\
\hline a. Chirauli & & & & \\
1 & Neem (57) & Bakaino (80) & Sal (80) & Sal (75) \\
2 & Dudhkaraiya (36) & Ipel-Ipel (75) & Bakaino (57) & Bakaino (38) \\
3 & Jamun (30) & Dumri (55) & Botdhairo (39) & Sisau (31) \\
4 & Bel (25) & Khannue (47) & Sisau (32) & Satisal (29) \\
5 & Med (22) & Panan (29) & Panan (22) & Panan (27) \\
b. Dhikurbari & Seem (32) & Sajan (28) & Sindure (46) & Sal (50) \\
1 & Hadchur (28) & Goleni (20) & Sal (21) & Bakaino (15) \\
2 & Dahikamle (18) & Khanue (18) & Baira (18) & Saj (12) \\
3 & Tulsi (17) & Lampate (15) & Piyal (17) & Gayo (10) \\
4 & Harro (10) & Gayo (12) & Botdhairo (13) & Jamun (8) \\
\hline
\end{tabular}

Figures in the parentheses are the total households reported the species. 
understanding of using native plant species. Since the several species used by one community differ from those used by another community, there is potential to repatriate planting materials and the knowledge from a place to another. The data also show that many people commonly use several species even though the species used in the two communities vary. More the commonly used for various purposes, higher might be the chance to be exploited and thus eroded. Therefore, many species will get endangered if initiative is not taken to conserve and utilize them. This necessitates detailed studies in the indigenous knowledge on morphology, habit and habitat, propagation methods, and the methods of use.

In addition to conservation efforts, the useful forest diversity needs to be promoted for the social, economic and ecological benefits in the communities. This is possible only with the active participation of all the concerned stakeholders including local communities, who rely on forest products. Involving community forest users groups in problem identification, planning, implementation and evaluation processes can enhance management practices in the participatory action. The locally available species have to be registered using locally available methods and technologies, which will be helpful for certification process in future. There are several multipurpose tree species that need more attention than any other species in order to meet multiple needs of households and communities from a few thriving species where reforestation or tree planting activities can not be introduced.

\section{Acknowledgements}

Seed Tree, Inc. is duly acknowledged for financial support to undertake this study.

\section{References Cited}

Khan, M.H. 1998. Documentation of indigenous knowledge in the Chepang community of Shaktikhor, VDC, Chitwan. In: K.K. Shrestha, P.K. Jha, P. Sheingji, A. Rastogi, S. Rajbhandary and M. Joshi (eds.), Ethnobotany for conservation and community development, Ethnobotanical Society of Nepal, Kathmandu, Nepal, 96-101.

Dangol, D.R. and S.B. Gurung. 1991. Ethnobotany of the Tharu tribe of Chitwan district, Nepal. Int. J. Pharmacognosy 29 (3): 203-209.

Dangol, D.R. 2002. Economic uses of forest plant resources in western Chitwan, Nepal. Banko Janakari 12(2): 56-64.

Manadhar, N.P. 1990. Folklore medicine of Chitwan district, Nepal. Ethnobotany 2: 31-38.

Muller-Boeker,U. 1993. Ethnobotanical studies among the Chitwan tharus. Journal Nepal Research Center 9: 17-56.

Rijal, A. 1994. Ethnobotany of Padampur: Analysis of dependency and conflict, M.Sc. thesis, Norwegian Center of International Agricultural Development. Agriculture University of Norway.

Shakya, S.M., D.R. Dangol and A. Shrivastava. 1995. Exploration of under exploited vegetables of Chitwan District, Nepal. Research report submitted to NEMP-IUCN, Nepal.69-92.

STN, 2002. Annual progress report: Integrated Human Ecology. Seed Tree Nepal, Chitwan, Nepal. 


\section{Appendix 1: Use of plant species in two communities}

\begin{tabular}{|c|c|c|c|c|c|c|c|c|c|c|}
\hline \multirow[b]{2}{*}{ S.N. } & \multirow[b]{2}{*}{ Nepali and Latin names } & \multirow[b]{2}{*}{ Habit } & \multicolumn{4}{|c|}{ Chirauli } & \multicolumn{4}{|c|}{ Dhikurbari } \\
\hline & & & Medicine & Timber & $\begin{array}{c}\text { Fuel } \\
\text { wood }\end{array}$ & Fodder & Medicine & Timber & $\begin{array}{c}\text { Fuel } \\
\text { wood }\end{array}$ & Fodder \\
\hline 1 & Amala, Phyllanthus emblica & $\mathrm{T}$ & $\mathrm{x}$ & & & & $\mathrm{x}$ & & & \\
\hline 2 & Amaro, Spondias pinnata & $\mathrm{T}$ & $\mathrm{x}$ & & & $\mathrm{x}$ & $\mathrm{x}$ & & & \\
\hline 3 & Amriso, Thysanolaena maxima & $\mathrm{H}$ & & & & $\mathrm{x}$ & & & & $\mathrm{x}$ \\
\hline 4 & Ank, Calatropis gigantea & S & $\mathrm{x}$ & & & & $\mathrm{x}$ & & & \\
\hline 5 & Ankhlejhar (Hadjorni), Equisetum debile & $\mathrm{H}$ & $\mathrm{x}$ & & & & & & & \\
\hline 6 & Anp, Mangifera indica & $\mathrm{T}$ & $\mathrm{x}$ & $\mathrm{x}$ & $\mathrm{x}$ & $\mathrm{x}$ & & & $\mathrm{x}$ & \\
\hline 7 & Archal, Antidesma acidum & $\mathrm{T}$ & & & & & & & $\mathrm{x}$ & $\mathrm{x}$ \\
\hline 8 & (Asidh) & $\mathrm{T}$ & $\mathrm{x}$ & & & & & & & \\
\hline 9 & Babari, Ocimum basilium & $\mathrm{H}$ & & & & & $\mathrm{x}$ & & & \\
\hline 10 & (Babin) & $\mathrm{T}$ & $\mathrm{x}$ & & & & & & & \\
\hline 11 & Babiyo, Eulaliopsis binnata & $\mathrm{H}$ & & & & $\mathrm{x}$ & & & & $\mathrm{x}$ \\
\hline 12 & (Babur), Acacia nilotica & $\mathrm{T}$ & $\mathrm{x}$ & & & & & & & \\
\hline 13 & Badahar, Artocarpus lakoocha & $\mathrm{T}$ & & $\mathrm{x}$ & $\mathrm{x}$ & $\mathrm{x}$ & & & & $\mathrm{x}$ \\
\hline 14 & Bakaino (Bakena), Melia azedarach & $\mathrm{T}$ & & $\mathrm{x}$ & $\mathrm{x}$ & $\mathrm{x}$ & & $\mathrm{x}$ & $\mathrm{x}$ & $\mathrm{x}$ \\
\hline 15 & Banjhi, Anogeissus latifolius & $\mathrm{T}$ & & & $\mathrm{x}$ & & & & $\mathrm{x}$ & \\
\hline 16 & Balu (Bariyar), Sida rhombifolia & $\mathrm{H}$ & $\mathrm{x}$ & & & & $\mathrm{x}$ & & & \\
\hline 17 & Bankera, Musa nepalensis & $\mathrm{H}$ & & & & $\mathrm{x}$ & & & & $\mathrm{x}$ \\
\hline 18 & Banso, Digitaria spp. & $\mathrm{H}$ & & & & $\mathrm{x}$ & & & & $\mathrm{x}$ \\
\hline 19 & Bar, Ficus benghalensis & $\mathrm{T}$ & & & & $\mathrm{x}$ & & & & \\
\hline 20 & Barro (Baraiya), Terminalia bellirica & $\mathrm{T}$ & $\mathrm{x}$ & & & & $\mathrm{x}$ & & & \\
\hline 21 & Bayar (Bahera), Zyziphus nummularia & $\mathrm{T}$ & $\mathrm{x}$ & & & & & & & \\
\hline 22 & Bel (Bel), Agle marmelos & $\mathrm{T}$ & $\mathrm{x}$ & & & & $\mathrm{x}$ & & & \\
\hline 23 & Betlauri, (Larkaiya), Costus speciosus & $\mathrm{H}$ & & & & & $\mathrm{x}$ & & & \\
\hline 24 & Bhalayo (Bhela), Semecarpus anacardium & $\mathrm{T}$ & & & $\mathrm{x}$ & & & & & \\
\hline 25 & (Bhatte) & $\mathrm{T}$ & & $\mathrm{x}$ & $\mathrm{x}$ & $\mathrm{x}$ & & & & \\
\hline 26 & Bhorla (Malhan), Baubinia vahlii & $\mathrm{C}$ & & & & $\mathrm{x}$ & & & & $\mathrm{x}$ \\
\hline 27 & Bhui amala, Phyllanthus urinaria & $\mathrm{H}$ & $\mathrm{x}$ & & & & $\mathrm{x}$ & & & \\
\hline 28 & Bojho (Bach), Acorus calamus & $\mathrm{H}$ & $\mathrm{x}$ & & & & $\mathrm{x}$ & & & \\
\hline 29 & Bot dhairo, Lagerstromia parviflora & $\mathrm{T}$ & & & $\mathrm{x}$ & & & & $\mathrm{x}$ & \\
\hline 30 & (Charari) & & $\mathrm{x}$ & & & & & & & \\
\hline 31 & Charchare lahara, Parthnocissus semicordata & $\mathrm{C}$ & & & & & & & & $\mathrm{x}$ \\
\hline 32 & Chiuri, Aesandra butyracea & $\mathrm{T}$ & $\mathrm{x}$ & & & & & & & \\
\hline 33 & Dabdabe (Jhengra), Garuga pinnata & $\mathrm{T}$ & & & & & & & & $\mathrm{x}$ \\
\hline 34 & Dahikamle (Dahigona), Callicarpa macrophylla & $\mathrm{H}$ & $\mathrm{x}$ & & & & $\mathrm{x}$ & & & \\
\hline 35 & Dalchini (Tejpat), Cinnamomum tamala & $\mathrm{T}$ & $\mathrm{x}$ & & & & $\mathrm{x}$ & & & \\
\hline 36 & Datiun, Achyranthes aspera & S & & & & & $\mathrm{x}$ & & & \\
\hline 37 & Dhairo (Dhaira), Woodfordia fruticosa & S & $\mathrm{x}$ & & $\mathrm{x}$ & & $\mathrm{x}$ & & $\mathrm{x}$ & $\mathrm{x}$ \\
\hline 38 & (Dhama) & & & & & $\mathrm{x}$ & & & & \\
\hline 39 & Dhupi, Thuja compacta & S & $\mathrm{x}$ & & & & & & & \\
\hline 40 & Dhurseli, Colebrookea oppositifolia & S & & & & & $\mathrm{x}$ & & & \\
\hline 41 & Dudhe lahar, Trachelospermum lucidum & $\mathrm{C}$ & & & & & & & & $\mathrm{x}$ \\
\hline 42 & Dumri (Dumari), Ficus racemosa & $\mathrm{T}$ & & & $x$ & $x$ & & & & $\mathrm{x}$ \\
\hline
\end{tabular}

Contd... 


\begin{tabular}{|c|c|c|c|c|c|c|c|c|c|c|}
\hline \multirow[b]{2}{*}{ S.N. } & \multirow[b]{2}{*}{ Nepali and Latin names } & \multirow[b]{2}{*}{ Habit } & \multicolumn{4}{|c|}{ Chirauli } & \multicolumn{4}{|c|}{ Dhikurbari } \\
\hline & & & Medicine & Timber & $\begin{array}{c}\text { Fuel } \\
\text { wood }\end{array}$ & Fodder & Medicine & Timber & $\begin{array}{c}\text { Fuel } \\
\text { wood }\end{array}$ & Fodder \\
\hline 43 & Galene, Leea crispa & $\mathrm{T}$ & & & & & & & & $\mathrm{x}$ \\
\hline 44 & Gandhe jhar, Ageratum conyzoides & $\mathrm{H}$ & $\mathrm{x}$ & & & & $\mathrm{x}$ & & & \\
\hline 45 & Gaujo, Milletia extensa & $\mathrm{S}$ & & & & & & & & $\mathrm{x}$ \\
\hline 46 & Gayo, Bridelia retusa & $\mathrm{T}$ & & & & & & $\mathrm{x}$ & $\mathrm{x}$ & $\mathrm{x}$ \\
\hline 47 & Ghiu kumari, Aloe vera & $\mathrm{H}$ & $\mathrm{x}$ & & & & $\mathrm{x}$ & & & \\
\hline 48 & Ghod tapre, Cassia tora & $\mathrm{H}$ & $\mathrm{x}$ & & & & $\mathrm{x}$ & & & \\
\hline 49 & Ginderi, Premna integrifolia & $\mathrm{T}$ & $\mathrm{x}$ & & & & & & & \\
\hline 50 & Gudurgano & $\mathrm{H}$ & $\mathrm{x}$ & & & & $\mathrm{x}$ & & & \\
\hline 51 & Gurjo ko lahara, Tinospora sinensis & $\mathrm{C}$ & $\mathrm{x}$ & & & & $\mathrm{x}$ & & & \\
\hline 52 & Gulmohar, Delonix regia & $\mathrm{T}$ & & & $\mathrm{x}$ & & & & & \\
\hline 53 & Hadchur (Med), Viscum album & $\mathrm{T}$ & $\mathrm{x}$ & & & & $\mathrm{x}$ & & & \\
\hline 54 & Hadebayar (Chhoti bayera), Zyziphus incurva & $\mathrm{S}$ & $\mathrm{x}$ & & & & & & & \\
\hline 55 & Harro (Haraiya), Terminalia chebula & $\mathrm{T}$ & $\mathrm{x}$ & & & & $\mathrm{x}$ & & & \\
\hline 56 & Imili, Tamarindus indica & $\mathrm{T}$ & $\mathrm{x}$ & & & & & & & \\
\hline 57 & Ipil Ipil, Laencoena leucocephala & $\mathrm{T}$ & & $\mathrm{x}$ & $\mathrm{x}$ & $\mathrm{x}$ & & $\mathrm{x}$ & & $\mathrm{x}$ \\
\hline 58 & Jamun (Jamu), Sysygium cumini & $\mathrm{T}$ & $\mathrm{x}$ & $\mathrm{x}$ & $\mathrm{x}$ & $\mathrm{x}$ & & $\mathrm{x}$ & $\mathrm{x}$ & $\mathrm{x}$ \\
\hline 59 & (Jhingad), Lannea coromadelica & $\mathrm{T}$ & $\mathrm{x}$ & & $\mathrm{x}$ & & & & & \\
\hline 60 & Kadam, Anthocephalus kadamba & $\mathrm{T}$ & & $\mathrm{x}$ & $\mathrm{x}$ & & & & & \\
\hline 61 & Kainyo, Wendlandia puberula & $\mathrm{T}$ & & & $\mathrm{x}$ & & & & & $\mathrm{x}$ \\
\hline 62 & Kalikath, Glochidion velutinum & $\mathrm{T}$ & & & $\mathrm{x}$ & & & & $\mathrm{x}$ & \\
\hline 63 & Kans (Jhaksi), Saccharum spontaneum & $\mathrm{H}$ & & & & $\mathrm{x}$ & & & & \\
\hline 64 & Kapok, Ceiba pentandra & $\mathrm{T}$ & & $\mathrm{x}$ & $\mathrm{x}$ & $\mathrm{x}$ & & & $\mathrm{x}$ & \\
\hline 65 & Karma (Haldu), Adina cordifolia & $\mathrm{T}$ & & $\mathrm{x}$ & $\mathrm{x}$ & $\mathrm{x}$ & & $\mathrm{x}$ & $\mathrm{x}$ & \\
\hline 66 & Katahar, Artocarpus heterophylla & $\mathrm{T}$ & & $\mathrm{x}$ & $\mathrm{x}$ & $\mathrm{x}$ & & $\mathrm{x}$ & & \\
\hline 67 & Khamari, Gmelia arborea & $\mathrm{T}$ & & & & & & & & $\mathrm{x}$ \\
\hline 68 & Khannue, Ficus semicordata & $\mathrm{T}$ & & & $\mathrm{x}$ & $\mathrm{x}$ & & & & $\mathrm{x}$ \\
\hline 69 & Khayar (Kattha), Acacia catechu & $\mathrm{T}$ & $\mathrm{x}$ & $\mathrm{x}$ & $\mathrm{x}$ & & & $\mathrm{x}$ & & \\
\hline 70 & Khirro (Dudhkaraiya), Holarrbena pubescens & $\mathrm{T}$ & $\mathrm{x}$ & & & & & & & \\
\hline 71 & (Khuja) & $\mathrm{T}$ & & & $\mathrm{x}$ & $\mathrm{x}$ & & & & \\
\hline 72 & Kimbu (Muslendi), Morus alba & $\mathrm{T}$ & & & & $\mathrm{x}$ & & & & $\mathrm{x}$ \\
\hline 73 & Koiralo (Koirala), Baubinia variegata & $\mathrm{T}$ & & & $\mathrm{x}$ & $\mathrm{x}$ & & & & $\mathrm{x}$ \\
\hline 74 & (Kumpadari) & $\mathrm{T}$ & & & $\mathrm{x}$ & & & & & \\
\hline 75 & Kumvi, Careya arborea & $\mathrm{T}$ & & & $\mathrm{x}$ & & & & & \\
\hline 76 & Kurilo (Khonta), Asparagus racemosus & $\mathrm{H}$ & $\mathrm{x}$ & & & & $\mathrm{x}$ & & & \\
\hline 77 & Kusum (Athera), Scleichiara oleosa & $\mathrm{T}$ & $\mathrm{x}$ & & $\mathrm{x}$ & $\mathrm{x}$ & & & & $\mathrm{x}$ \\
\hline 78 & Kutmiro, Litsea monopetala & $\mathrm{T}$ & & & $\mathrm{x}$ & $\mathrm{x}$ & & & $\mathrm{x}$ & $\mathrm{x}$ \\
\hline 79 & Kyamun (Kyamuna), Cleistocalyx operculatus & $\mathrm{T}$ & & & & & $\mathrm{x}$ & & & \\
\hline 80 & Lajjawati (Lajapati), Mimosa pudica & $\mathrm{H}$ & $\mathrm{x}$ & & & & & & & $\mathrm{x}$ \\
\hline 81 & Lampate, Aesculus indica & $\mathrm{T}$ & & & & & & & & $\mathrm{x}$ \\
\hline 82 & Lankuri, Fraxinus floribunda & $\mathrm{T}$ & & & & $\mathrm{x}$ & & & & $\mathrm{x}$ \\
\hline 83 & Latikath, Swida oblonga & $\mathrm{T}$ & & & $\mathrm{x}$ & & & & & \\
\hline 84 & Lunde kanda, Amaranthus spinosa & $\mathrm{H}$ & $\mathrm{x}$ & & & & $\mathrm{x}$ & & & \\
\hline 85 & Main kanda, Xeromphis spinosa & $\mathrm{S}$ & $\mathrm{x}$ & & & & & & & \\
\hline
\end{tabular}

Contd... 


\begin{tabular}{|c|c|c|c|c|c|c|c|c|c|c|}
\hline \multirow[b]{2}{*}{ S.N. } & \multirow[b]{2}{*}{ Nepali and Latin names } & \multirow[b]{2}{*}{ Habit } & \multicolumn{4}{|c|}{ Chirauli } & \multicolumn{4}{|c|}{ Dhikurbari } \\
\hline & & & Medicine & Timber & $\begin{array}{c}\text { Fuel } \\
\text { wood }\end{array}$ & Fodder & Medicine & Timber & $\begin{array}{c}\text { Fuel } \\
\text { wood }\end{array}$ & Fodder \\
\hline 86 & (Morthaiya), Deeringia celisiodes & $\mathrm{T}$ & $\mathrm{x}$ & & & & & & & \\
\hline 87 & Mothe (Motha), Cyperus rotundus & $\mathrm{H}$ & & & & $\mathrm{x}$ & & & & $\mathrm{x}$ \\
\hline 88 & Neem (Nimi), Azadirachta indica & $\mathrm{T}$ & $\mathrm{x}$ & & & & $\mathrm{x}$ & & & \\
\hline 89 & Nimaro (Pakadi), Ficus roxbeurghii & $\mathrm{T}$ & $\mathrm{x}$ & & $\mathrm{x}$ & $\mathrm{x}$ & & & & $\mathrm{x}$ \\
\hline 90 & Palans, Butea monosperma & $\mathrm{T}$ & & & & $\mathrm{x}$ & & & & $\mathrm{x}$ \\
\hline 91 & Patpate, Physalis divaricata & $\mathrm{H}$ & & & & $\mathrm{x}$ & & & & \\
\hline 92 & Pharsa, Grewia pumila & $\mathrm{S}$ & & & & $\mathrm{x}$ & & & & \\
\hline 93 & Pidalu (Kachu), Colocasia antiquorum & $\mathrm{H}$ & $\mathrm{x}$ & & & & & & & \\
\hline 94 & Pidar, Xeromphis ulignosa & $\mathrm{T}$ & $\mathrm{x}$ & & & & & & & \\
\hline 95 & Pipal (Pipar), Ficus religiosa & $\mathrm{T}$ & $\mathrm{x}$ & & $\mathrm{x}$ & $\mathrm{x}$ & & & & \\
\hline 96 & Pipla (Pipari), Piper longum & $\mathrm{H}$ & $\mathrm{x}$ & & & & $\mathrm{x}$ & & & \\
\hline 97 & Piyal & $\mathrm{T}$ & & & & & & & $\mathrm{x}$ & \\
\hline 98 & Rajbrikchhya (Ahiroga), Cassia fistula & $\mathrm{T}$ & $\mathrm{x}$ & & $\mathrm{x}$ & & $\mathrm{x}$ & & & \\
\hline 99 & Rittha, Sapindus mukorossi & $\mathrm{T}$ & & & & & $\mathrm{x}$ & & & \\
\hline 100 & Rudilo (Dlehi bhanthi), Pogostemon benghalensis & S & & & & & $\mathrm{x}$ & & & $\mathrm{x}$ \\
\hline 101 & (Sahoroti) & $\mathrm{T}$ & $\mathrm{x}$ & & $\mathrm{x}$ & & & & & \\
\hline 102 & Saj (Asna), Terminalia alata & $\mathrm{T}$ & & $\mathrm{x}$ & $\mathrm{x}$ & & & $\mathrm{x}$ & & \\
\hline 103 & Sajan (Panwan), Desmodium oojeinense & $\mathrm{T}$ & & $\mathrm{x}$ & $\mathrm{x}$ & $\mathrm{x}$ & & $\mathrm{x}$ & & \\
\hline 104 & Sal (Sekhuwa), Shorea robusta & $\mathrm{T}$ & & $\mathrm{x}$ & $\mathrm{x}$ & & & $\mathrm{x}$ & $\mathrm{x}$ & $\mathrm{x}$ \\
\hline 105 & Sal lahara, Spatholobus parviflorus & $\mathrm{C}$ & & & & & & & & $\mathrm{x}$ \\
\hline 106 & Sarpagandha (Dharmaruwa), Rauvolfia serpentina & $\mathrm{H}$ & $\mathrm{x}$ & & & & & & & \\
\hline 107 & Satisal, Dalbergia latifolia & $\mathrm{T}$ & & $\mathrm{x}$ & $\mathrm{x}$ & & & $\mathrm{x}$ & & \\
\hline 108 & Satuwa, Paris polyphylla & $\mathrm{T}$ & & & & & $\mathrm{x}$ & & & \\
\hline 109 & (Sehuli) & S & & & & $\mathrm{x}$ & & & & \\
\hline 110 & Simal (Simar), Bombax ceiba & $\mathrm{T}$ & & $\mathrm{x}$ & $\mathrm{x}$ & $\mathrm{x}$ & & & & \\
\hline 111 & Sindure (Roeni), Mallotus phillippensis & $\mathrm{T}$ & & & $\mathrm{x}$ & & & & $\mathrm{x}$ & $\mathrm{x}$ \\
\hline 112 & (Sinuri) & & & & $\mathrm{x}$ & & & & & \\
\hline 113 & Siris Alberia spp. & $\mathrm{T}$ & & & $\mathrm{x}$ & $\mathrm{x}$ & & & & \\
\hline 114 & Siru, Imperata cylindrica & $\mathrm{H}$ & & & & $\mathrm{x}$ & & & & $\mathrm{x}$ \\
\hline 115 & Sisoo (Sisuwa), Dalbergia sisoo & $\mathrm{T}$ & & $\mathrm{x}$ & $\mathrm{x}$ & & & $\mathrm{x}$ & & \\
\hline 116 & Sitalchini (Sohajan), Moringa oleifera & $\mathrm{T}$ & $\mathrm{x}$ & & & & & & & \\
\hline 117 & Tanki, Baubinia purpurea & $\mathrm{T}$ & & & $\mathrm{x}$ & $\mathrm{x}$ & & & & $\mathrm{x}$ \\
\hline 118 & Tatelo (Patsan) Oroxylum indicum & $\mathrm{T}$ & $\mathrm{x}$ & & $\mathrm{x}$ & $\mathrm{x}$ & & & & $\mathrm{x}$ \\
\hline 119 & Tantari (Tetari), Dillenia pentagyna & $\mathrm{T}$ & $\mathrm{x}$ & & & $\mathrm{x}$ & & & & $\mathrm{x}$ \\
\hline 120 & Tapre, Cassia tora & $\mathrm{H}$ & $\mathrm{x}$ & & & & & & & \\
\hline 121 & Teek (Sawan), Tectona grandis & $\mathrm{T}$ & & $\mathrm{x}$ & & $\mathrm{x}$ & & & & \\
\hline 122 & Thakal (Khajurati), Phoenix bumilis & $\mathrm{H}$ & & & & $\mathrm{x}$ & & & & \\
\hline 123 & Thotne (Kothaiya), Ficus hispida & $\mathrm{T}$ & & & $\mathrm{x}$ & $\mathrm{x}$ & & & & $\mathrm{x}$ \\
\hline 124 & Timur, Zanthozylum armatum & $\mathrm{H}$ & & & & & $\mathrm{x}$ & & & \\
\hline 125 & Titepati (Pati), Artemisia dubea & $\mathrm{H}$ & $\mathrm{x}$ & & & & $\mathrm{x}$ & & & \\
\hline 126 & Tulsi, Ocimum tenuiflorum & $\mathrm{H}$ & $\mathrm{x}$ & & & & $\mathrm{x}$ & & & \\
\hline 127 & (Uchharinga) & & $\mathrm{x}$ & & & & & & & \\
\hline 128 & (Vellar), Trewia nudiflora & $\mathrm{T}$ & & & $\mathrm{x}$ & & & & & \\
\hline & Total & & 56 & 18 & 45 & 43 & 34 & 12 & 15 & 40 \\
\hline
\end{tabular}

Word in parenthesis indicate Tharu name; $\mathrm{x}$ indicates species use. 